\title{
Tracing Mental Models in Cartographic Datasets -The Case of OpenStreetMap
}

\author{
Franz-Benjamin Mocnik \\ University of Twente, Franz-Benjamin Mocnik-franz-benjamin.mocnik@utwente.nl
}

Keywords: collaboration, cartographic representation, place, subjectvity, shared data sources, OpenStreetMap (OSM)

\begin{abstract}
:
Geographical features can be represented in different ways. Buildings, for instance, can be represented as areal features defined by polygonal lines or as point features in a map. While the type of representation chosen to represent a building strongly depends on the scale of the map, it seems common to represent points of interests (POIs) as point features. More complex examples exist. For example, the way buildings that are part of a mosque are conceptualized and thus labelled in a map strongly depends on how familiar we are with the Arabic culture. The same applies to the often perceived separation between public and private space in Arabic cultures, which can have an impact on geometrical aspects of map representations. Such coexisting representations of geographical features can, in particular, be observed in collaboratively created data sources (Mocnik et al., 2019), such as OpenStreetMap (OSM) data and maps generated from these.
\end{abstract}

Competing representations of geographical features can reduce the readability of a map, but they can also open up opportunities to draw conclusions about the underlaying mental model (Mayer et al., 2020). When the people involved in the map creation process have different mental representations of a geographical feature, the representations they create often differ. This, in turn, impacts the readability, which refers to the process of transferring a map representation into a mental one, i.e., the process opposed to map creation, as there is often no clear and unambiguous correspondence between the symbols in the map and the represented geographical features (Scheider et al., 2009; Mocnik et al., 2018) Over time, these conceptualizations can mutually influence and lead to a convergence (Mocnik et al., 2017). Despite this, the coexistence of different cartographic representations makes possible to draw conclusions about the way a feature has been conceptualized. The symbols in the map encode, accordingly, besides the intended information about the geographical features, also information about the mental models involved (Mayer et al., 2020).

The effect of mental models on the map representation often goes unnoticed because there is, in many cases, only very subtle variation between the map representations of one type of geographical features. If such variation would be much larger, it would even be impossible to interpret the symbols of a map. While the examples discussed above can be traced very well in individual cases, it is yet unclear how systematic the influence of mental models is. A systematic examination of such examples can investigate the influence statistically and at a larger scale. Further research might show the extent to which conclusions can practically be drawn about the conceptualization of geographical features and corresponding mental representations.

The ability to trace mental models through maps is a suitable tool for exploring the conceptualization of geographies. For instance, future research might investigate in detail the impact culture has on the way we conceptualize. Likewise, it might investigate how people experience urban landscapes in different ways. By using maps and the data behind them as a source of information for mental models, maps gain a new purpose while the original one is put into the background. While such thinking is common for sketch maps, it also also applies, to a lesser degree, to Volunteered Geographic Information (VGI), which can become a valuable data source for ethnologists, human geographers, psychologists, and cognitive scientists.

In a similar way, a better understanding of how mental models relate to cartographic representations can help to improve the latter. The cartographic representation of idiosyncratically experienced geographies, for instance, still poses challenges. Also, stories are, in many cases, hard to convey through classical maps (Mocnik and Fairbairn, 2018). The same applies to places and corresponding cartographic representations, which often are characterized by subjective and idiosyncratic aspects (Westerholt et al., 2018; Westerholt et al., 2020). The empirical investigation of the effect mental models have on cartographic representations can be expected to also provide insights into the reverse process, thus leading to cartographic techniques that make it possible to convey idiosyncratic experience and, eventually, also emotions. 


\section{Acknowledgements}

The author expresses his sincere thanks to Daniel W. Heck for fruitful discussions on collaboration in the context of Psychology. This work has been supported by the project 'Heterogeneity and Convergence in Shared Data Sources The Importance of Cognitive Coherence in Collective Decision Making' funded by the Heidelberg Academy of Sciences and Humanities.

\section{References}

Mayer, M., Heck, D.W. and Mocnik, F.-B., 2020. Shared Mental Models as a Psychological Explanation for Converging Mental Representations of Place - The Example of OpenStreetMap. In: Mocnik, F.-B. and Westerholt, R. (eds.). Proceedings of the 2nd International Symposium on Platial Information Science (PLATIAL'19), pp. 43-50. doi:10.5281/zenodo.3628871

Mocnik, F.-B., Zipf, A. and Raifer, M., 2017. The OpenStreetMap Folksonomy and Its Evolution. Geo-spatial Information Science 20(3), pp. 219-230. doi:10.1080/10095020.2017.1368193

Mocnik, F.-B. and Fairbairn, David, 2018. Maps Telling Stories? The Cartographic Journal 55(1), pp. 36-57. doi:10.1080/00087041.2017.1304498

Mocnik, F.-B., Mobasheri, A., Griesbaum, L., Eckle, M., Jacobs, C. and Klonner, C., 2018. A Grounding-Based Ontology of Data Quality Measures. Journal of Spatial Information Science 16, pp. 1-25. doi:10.5311/JOSIS.2018. 16.360

Mocnik, F.-B., Ludwig, C., Grinberger, A.Y., Jacobs, C., Klonner, C. and Raifer, M., 2019. Shared Data Sources in the Geographical Domain-A Classification Schema and Corresponding Visualization Techniques. ISPRS International Journal of Geo-Information 8(5), 242. doi:10.3390/ijgi8050242

Scheider, S., Janowicz, K. and Kuhn, W., 2009. Grounding Geographic Categories in the Meaningful Environment. Proceedings of the 9th Conference on Spatial Information Theory (COSIT), pp. 69-87. doi:10.1007/978-3-642-038327_5

Westerholt, R., Gröbe, M., Zipf, A. and Burghardt, D., 2018. Towards the Statistical Analysis and Visualization of Places. Proceedings of the 10th International Conference on Geographic Information Science (GIScience), pp. 63:163:7. doi:10.4230/LIPIcs.GISCIENCE.2018.63

Westerholt, R., Mocnik, F.-B. and Comber, Alexis, 2020. A Place for Place: Modelling and Analysing Platial Representations. Transactions in GIS 24(4), pp. 811-818. doi:10.1111/tgis.12647 